\title{
COMBINED AGAR DIFFUSION AND REPLICA PLATING TECHNIQUES IN THE STUDY OF ANTIBACTERIAL SUBSTANCES
}

\author{
BY \\ S. D. ELEK AND G. R. F. HILSON \\ From the Department of Bacteriology, St. George's Hospital Medical School, London
}

(RECEIVED FOR PUblication AUgUST 7, 1953)

Much interest has recently been aroused in the field of antibiotics by two distinct but related problems. The first concerns the possibility that in certain types of infection, notably subacute bacterial endocarditis, the bactericidal action of a drug is of greater therapeutic significance than the inhibitory effect (Hunter 1950; Robbins and Tompsett, 1951 ; Cates, Christie, and Garrod, 1951). The second problem is the action of antibiotics used in combination. In this respect the findings of many workers indicate that different mixtures may show augmented or diminished bactericidal effect, and the result may be influenced by the species or strain of organism tested (Jawetz, Gunnison, Bruff, and Coleman 1952 ; Bliss, Warth, and Long, 1952).

Any investigation of these two problems requires a method applicable to routine use for the assessment of the bactericidal action of antibiotics. None of the conventional methods used for these purposes is suitable for large-scale application, as they depend on some form of viable counting and are very laborious. Even the more convenient turbidimetric technique described by Burnell and Kirby (1951) is too elaborate for general use. Moreover, a disadvantage common to all these methods is that an appreciable amount of drug is inevitably transferred with any sample to the new medium used to measure the bactericidal action. If the proportion of surviving bacteria is low it may be necessary either to limit the range of concentration of the drug under study, or to destroy it in some way which does not affect the bacteria. This is not always possible or convenient. A method of transfer which carries over little more than the amount of drug adhering to individual organisms is therefore needed. The method described here, which is an application of the technique of "replica plating" described by Lederberg and Lederberg (1952) and used by them for studying problems of bacterial genetics, is believed to fulfil these requirements. It involves no significant transfer of drug from one medium to another, is simple enough for routine use, and may be applied as a continuation of a form of " sensitivity test" in common use. It consists essentially of the transfer of bacteria from one nutrient agar surface to another without disturbing their spatial relationships. The transferring agent is the pile of a piece of sterile velvet covering a flat surface. The success of the technique depends on the fact that moisture is absorbed by the pile and no lateral smearing of the impression occurs.

\section{Materials}

Construction of the "Stamps."-Discs, $3 \mathrm{~cm}$. thick and of a diameter slightly less than that of the Petri dish with which they are to be used, are made from well-seasoned, close-grained wood. The parallel faces are "band-faced" to give a smooth plane surface. An even layer of " copydex" latex adhesive sufficiently thick to hide the grain of the wood is rapidly brushed over one face and a piece of velvet is smoothed on to it with the pile facing away from the wood. After about two minutes the edge of the velvet is trimmed to the size of the disc. Good-quality furnishing velour of about $2.5 \mathrm{~mm}$. thickness with a close pile has been found suitable: dress material is unsatisfactory. A set of stamps so prepared is sterilized by autoclaving: any which become warped as a result have to be discarded. Pairs of stamps with the velvet faces in contact can be stored ready for use. One stamp may be used many times, but if its velvet surface becomes matted or uneven, the fabric should be replaced.

Primary Plates.-Nutrient media must be firm enough to withstand moderate pressure without splitting: New Zealand agar in $2 \%$ concentration has proved suitable. Plates are dried at $37^{\circ} \mathrm{C}$. for two hours and the surface inoculated by flooding with young broth cultures just beginning to show turbidity. The inoculum density should be such as to give just 


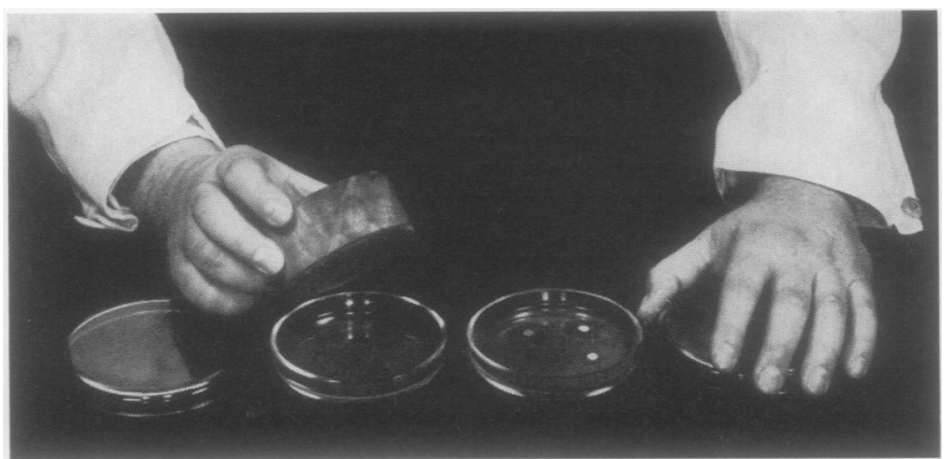

FIG. 1.-The use of the velvet pad in making a replica of a routine " sensitivity plate."

agar plates were flooded with each. After drying replica transfers were made with small stamps $13 \mathrm{~cm}$. square) which left more than half of the surface of the primary plate undisturbed. Primary and replica plates were incubated and the resulting growths compared (Fig. 2). It is evident that in the range of incoulum employed, approximately one organism out of a hundred has been transferred to the replica plate. For more accurate assessment, the number of colonies in a sample area of 4 sq. $\mathrm{cm}$. was counted on the plates carrying colonies sufficiently dispersed. The experiment was repeated

confluent growth. Excess fluid is pipetted off and if plates have been inoculated while still warm from the incubator they are found to dry off within five minutes.

Replica Plates.-These also contain $2 \%$ agar and the nutrient base most suited for the development of the particular bacteria under test.

\section{Experimental}

Stamping.-The velvet surface of a sterilized stamp is pressed evenly and firmly against the primary plate, avoiding lateral movement. The pad is then lifted off and applied in a similar way to the replica plate (Fig. 1). Alternatively, each plate may be pressed in turn against the upturned surface of a stamp.

Proportion of Organisms Transferred by Replica Plating.-For quantitative interpretation of the results the fraction of the total viable population transferred by the velvet must be known. This was estimated in the following ways:-(1) Serial tenfold dilutions were prepared from a young broth culture and nutrient three times with a staphylococcus as test organism and three times with a coliform bacillus. The results showed a transfer of between 1 in 60 and 1 in 27 (average 1 in 40 ) of the organisms on the primary plate. It was also estimated that the surface inoculation produced by flooding a plate with an undiluted young broth culture was of the order of 5 million bacteria per sq. $\mathrm{cm}$.

(2) The transfer factor of about 1 in 40 obtained by the method described was of necessity determined from primary plates carrying not more than a few hundred bacteria per sq. $\mathrm{cm}$. It seemed possible that a much smaller fraction of organisms might be transferred from an area bearing several millions of bacteria per sq. $\mathrm{cm}$., and it was important to determine this directly, since this was the inoculum density chosen for the subsequent experiments. The method of Burnell and Kirby (1951) was found to be suitable for this purpose. Primary and replica plates of serial tenfold dilutions of a broth culture were prepared in the manner just described. As soon as the plates

FIG. 2.-An illustration of the proportion of organisms transferred by replica plating. The upper row shows the growth on primary plates inoculated with serial tenfold dilutions of a staphylococcal broth culture. Replica samples were taken from each before incubation, and these are shown in the lower row. 
had dried a sample was taken from each plate by punching out a disc of agar with a cork-borer $1 \mathrm{~cm}$. in diameter. Each disc was dropped into a test-tube $(150 \mathrm{~mm}$. $\times 16 \mathrm{~mm}$.) containing $5 \mathrm{ml}$. of broth; the tubes were shaken vigorousiy at once and incubated in a water-bath at $37^{\circ} \mathrm{C}$. The development of turbidity in the tubes was recorded at 20 -min. intervals in an absorptiometer. Turbidity curves were plotted for each tube, the point of $50 \%$ maximum turbidity determined for each curve, and a note made of the time taken for this degree of turbidity to be reached. Burnell and Kirby showed that this value is proportional to the number of viable organisms inoculated into a tube. The results of this experiment indicated that the sample disc of agar from a primary plate carried roughly one hundred times as many organisms as did the sample from the corresponding replica plate. This relationship was found with the largest inoculum and its first dilution. There is therefore good agreement between the two methods used in estimating the proportion of organisms transferred by replica plating. It is of the order of 1 in 100 with the density of inocula used.

\section{Estimation of Bactericidal Action}

Method 1.-In this procedure predetermined amounts of the drug to be tested are incorporated evenly in the medium and replica samples are taken to record its lethal action. Primary plates are poured with a series of increasing dilutions of the drug and inoculated in the way described. Replica samples are taken from each plate at intervals during incubation using stamps $3 \mathrm{~cm}$. square. As many as 12 samples can be taken from a single large plate $(14 \mathrm{~cm}$. in diameter). In this way it is possible to determine the rate at which the different concentrations of drug in each primary plate prove lethal. Although this method is less accurate than a series of viable counts, it may find a useful application in the testing of antiseptics. These are usually used in relatively high concentration and it is notoriously difficult to eliminate errors due to the transference of antiseptics; the replica method is free from this objection.

Method 2.-This procedure is more suitable for routine use with antibiotics. A "sensitivity plate" is prepared according to the paper disc method of Fairbrother and Martyn (1951), using a young broth culture of the organism to be tested. It may be convenient to test the action of up to four antibiotics on one $9 \mathrm{~cm}$. Petri dish. The concentration of antibiotic with which a paper disc is soaked should be about 20 times as great as the maximum concentration which can be achieved in the body by normal therapeutic dosage, since the diffusion of the drug into a layer of agar $4 \mathrm{~mm}$.

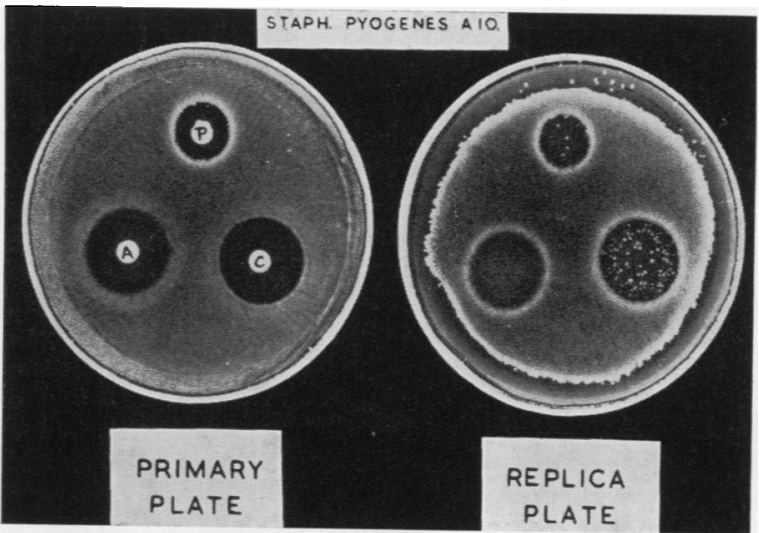

FIG. 3.-A " sensitivity plate" and its replica. The organism is a coagulase-positive staphylococcus. $\mathrm{P}$ : penicillin; A: aureo-
mycin; C: chloramphenicol.

deep (the usual depth) produces a dilution of about 1 in 20 in the medium underlying the paper disc. In practice, such concentrations will produce zones of inhibition of a sensitive organism of 15 to $20 \mathrm{~mm}$. diameter after overnight incubation. The zones produced by each antibiotic are measured. A replica plate is made and incubated. An example of a primary plate and its replica is shown in Fig. 3. The lethal action of aureomycin and penicillin on the staphylococcal inoculum has been virtually complete and the diameter of its zone is as large as that of the inhibition zone on the primary plate. The zone of lethal action of chloramphenicol is also equal in size to its inhibitory zone but the bactericidal effect is markedly less than that of the other two antibiotics.

The degree of lethal action may be estimated by comparing the size of the replica zone with that of the inhibition zone and by comparing the colony density within it with a reference chart similar to Fig. 2. The bactericidal effect is recorded as + when no more than a few colonies develop within the zone (approximately 1 in 10,000 survival) and \pm for more crowded, discrete colonies. Semi-confluent or greater growth is taken to indicate no significant bactericidal effect. For example, the lethal effect of aureomycin and penicillin in Fig. 3 would be recorded as + while that of chloramphenicol would be \pm . Thus a simple visual assessment is afforded of the bactericidal action of a drug on a given strain.

This method has been used to test the action of seven antibiotics against 65 strains of bacteria of different species. The detailed results will be reported later, but certain aspects of interpretation which arose during the investigation must be 


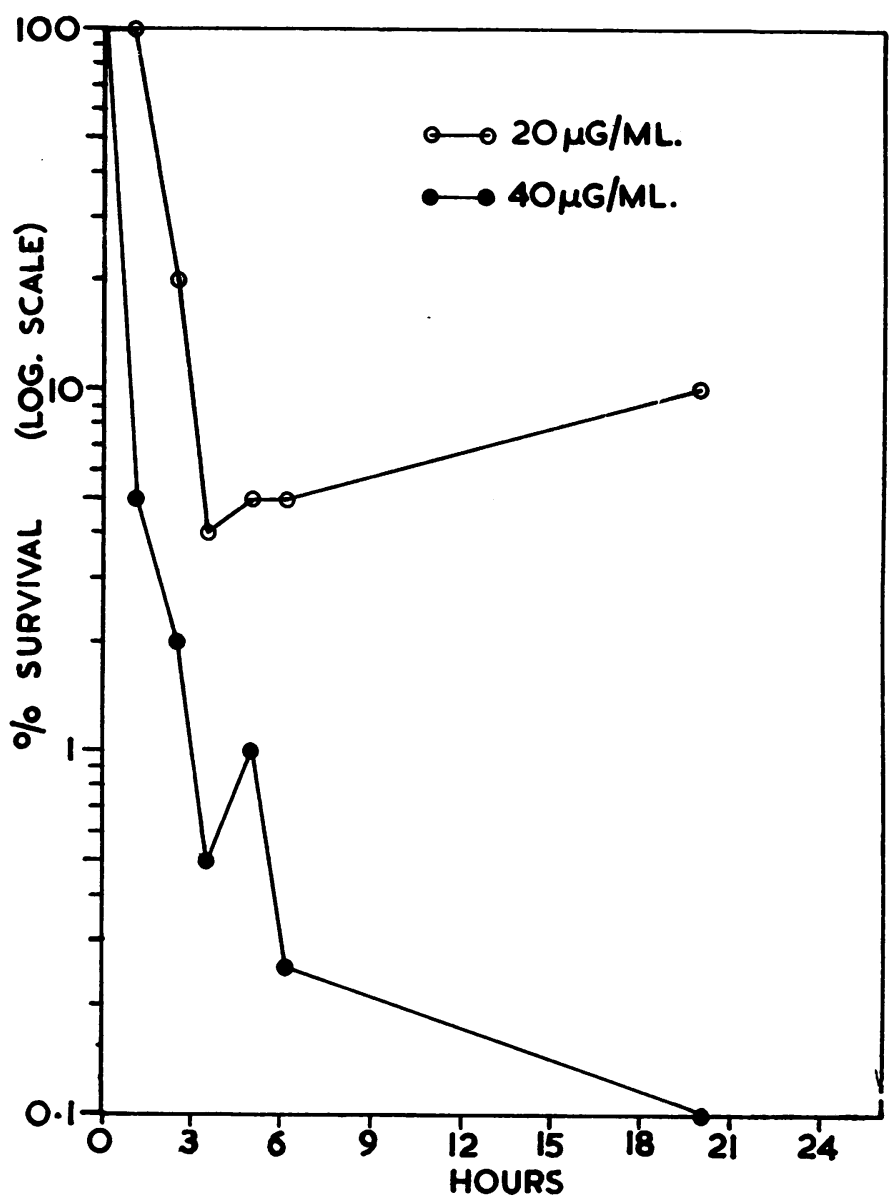

Fig. 4.-The lethal effect of different concentrations of chloramphenicol on a strain of Bact. coli.

pointed out. In considering them the dynamic nature of the diffusion system should be borne in mind. Humphrey and Lightbown (1952) have shown that with the concentrations used the amount of antibiotic rises near the central disc during the first hour to a maximum of some 10 or 15 times the minimum inhibitory concentration. It then diminishes steadily as outward diffusion occurs until at 20 hours it has fallen to a value of about four times the minimum inhibitory concentration. Experience has shown that the boundary of the zone of inhibition becomes established after about four to five hours' incubation, and it may be concluded that the minimum intibitory concentration is attained at this time. The concentration of antibiotic in this area rises slightly further and is then maintained for 20 hours or more. With most drugs, of course, the zone in which the minimum inhibitory concentration is reached continues to expand but its $\Rightarrow$ influence passes unnoticed since growth $\stackrel{0}{\rightarrow}$ of the organisms has already taken? place. If, however, the minimum inhibitory concentration is also rapidly $\frac{\bar{\sigma}}{\partial}$ lethal, the organisms lying just outside $\stackrel{\mathbb{Q}}{\varrho}$ the boundary of the inhibition zone are killed: this is readily demonstrated $\overrightarrow{.}$

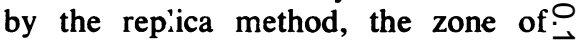
lethal effect being then larger than the $\vec{\omega}$

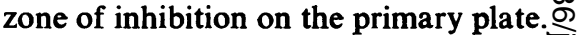
The expansion of the zone of mini- $\frac{0}{8}$ mum inhibitory concentration does $-V$ not always occur. Humphrey and $\vec{\omega}$ Lightbown (1952) have found that $v$ when streptomycin is tested against $\frac{\text { ? }}{5}$ Staph. aureus there is a very sharpdecrease in the concentration of the $\mathbb{\Phi}_{\mathbb{D}}$ drug just outside the boundary of the inhibition zone. They believe that this is due to the adsorption of streptomycin to these Gram-positive $\vec{\oplus}$ organisms preventing its effective $\perp$ concentration from reaching the minimum inhibitory level beyond the boundary. It is clear that the lethal effect detected by the replica method will not then have a zone size greater $\mathbb{D}$ than the zone of inhibition. A third $\stackrel{2}{\rightleftharpoons}$ possibility is that the size of the zone $\frac{\rho}{3}$ of lethal effect is considerably smaller $\bar{\sigma}$ than the inhibitory zone on the primary항 plate. This has in practice been found to occur with many antibiotics and organisms tested. It is interpreted as indicating that a concentration several times higher than the minimum inhibitory concen- $O$ tration is necessary to kill the bacteria within the time during which the primary plate is incubated, 음 and that such a concentration is reached only $D$ towards the centre of the area of diffusion. Confirmation for this interpretation is afforded by the $N$ results of the following experiment:

A strain of Bact. coli and chloramphenicol were $\stackrel{N}{\circ}$ chosen because they gave a wide difference between $\mathrm{\omega}$ the zone of inhibition on the primary plate (dia-O meter $25 \mathrm{~mm}$.) and the zone of lethal effect on theo rep'ica plate (diameter only $13 \mathrm{~mm}$.). The rates $\Phi$ of lethal action of different concentrations of the $\stackrel{?}{+}$ drug were tested by Method 1 . The concentrations $\frac{T}{\circ}$ of chloramphenicol used were $80,40,20,10,5$, and $\frac{P}{\mathbb{D}}$ $2.5 \mu \mathrm{g}$. per ml. Replica samples were taken at $20-\stackrel{\varrho}{\odot}$ minute intervals for the first hour, then at hourly $\stackrel{\mathbb{Q}}{\varrho}$ intervals for five hours, and finally after 20 hours. O The minimum inhibitory concentration in this 0 
titration was taken as $10 \mu \mathrm{g}$. per ml. since no inhibition occurred with the lower concentrations. The results with $40 \mu \mathrm{g}$. and $20 \mu \mathrm{g}$. per ml. are shown graphically in Fig. 4. From this it can be seen that twice the minimum inhibitory concentration produced only a $90 \%$ reduction of viable organisms after 20 hours, while four times the minimum inhibitory concentration killed $99 \%$ within five hours and $99.9 \%$ within 20 hours. These findings support the view that if the zone of lethal action is markedly smaller than the inhibition zone, the minimum lethal concentration of the antibiotic for the organism tested is significantly higher than the minimum inhibitory concentration.

\section{Antibiotic Interactions}

The demonstration of interactions between antibiotics becomes a relatively simple matter with the technique of agar diffusion combined with replica plating, and can be carried out in a number of ways.

Method 3: "Incorporation and Diffusion System."-This method is an extension of that described in Method 1 above for the estimation of bactericidal effect. A nutrient agar plate is poured with medium containing one drug, usually in a concentration which is just high enough to inhibit the growth of the inoculum. The plate is flooded with a young broth culture and dried rapidly. A blotting paper disc containing the second antibiotic is placed on it without delay. The plate is incubated for any chosen period of time and a replica plate made. The zone in which the organisms have been exposed to the action of both antibiotics is then examined for any difference in the rate of survival compared with the rest of the plate. To give an example, an experiment was performed in which a concentration of chloramphenicol just inhibitory to the staphylococcal inoculum was contained in the primary plate. Blotting paper discs soaked in penicillin, 1, 10, and 100 units per $\mathrm{ml}$, were placed on its surface and the plate incubated overnight. The replica plate made from this is illustrated in Fig. 5, from which it will be seen that over the plate in general there is a moderate degree of survival. However, in the zones where penicillin has also acted on the organisms (which were fully penicillin-sensitive by ordinary testing) the lethal effect of the chloramphenicol is almost completely prevented, showing that there is antagonism between these two antibiotics with this organism. The diameter of the effect is proportional to the original concentration

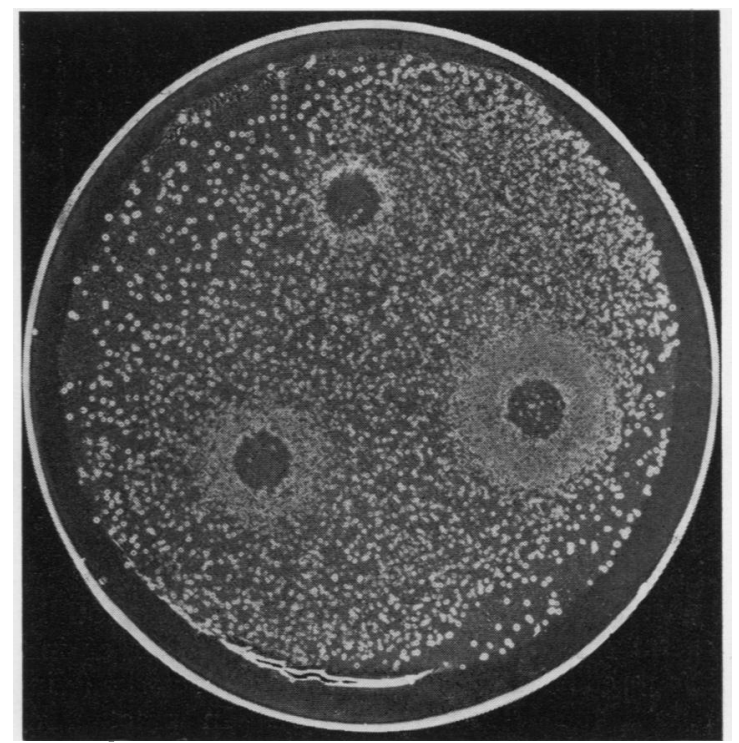

FIG. 5.-A replica plate illustrating antagonism by penicillin of the lethal effect of chloramphenicol on a coagulase-positive staphylococcus. Disc concentrations of penicillin on the primary plate: upper zone, $1 \mathrm{u} . / \mathrm{ml}$.; lower left, $10 \mathrm{u}$. $\mathrm{ml}$.; lower right, 100 $\mathrm{u} . \mathrm{ml}$. Chloramphenicol concentration in the primary plate: $15 \mu \mathrm{g} . / \mathrm{ml}$.

of penicillin, and it is clear that the antagonism is not reversed by relatively high concentrations of penicillin.

Method 4 : Paper-strips at Right Angles.-This is an application of a technique originally used for serological analysis (Elek, 1949). The principle involved is shown diagrammatically in Fig. 6. When strips saturated with a diffusible substance are placed at right angles to each other on the surface of a plate all combinations of concentrations occur as a result of diffusion in the area

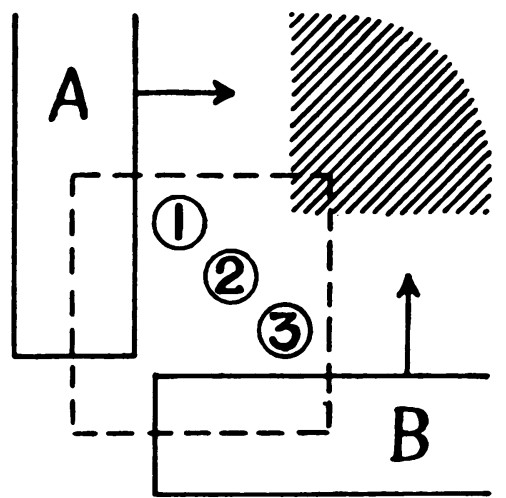

FIG. 6.-Diagram representing the result of diffusion of two antibiotics from paper strips at right angles. At point (1) drug $\mathbf{A}$ will be in high concentration, and $B$ in low concentration; at (2) both will be in moderate concentration; at (3) the reverse of (1) exists. The area of shading represents bacterial growth, with inhibition by both drugs. 


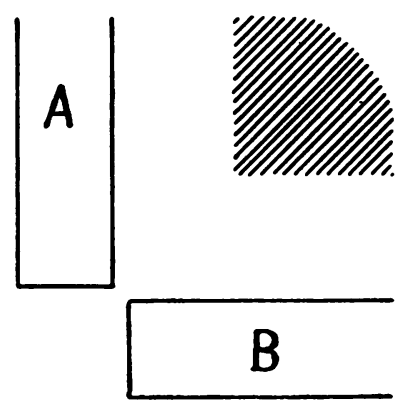

\section{No interference}

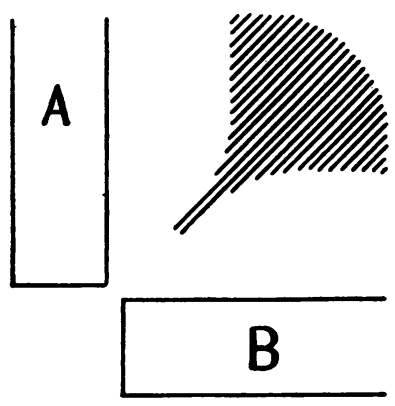

Antagonism

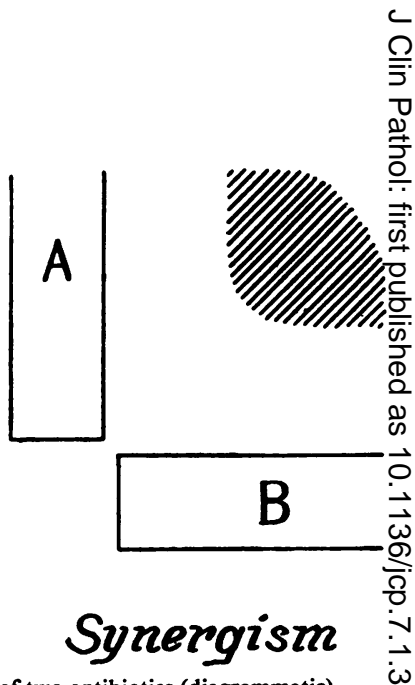

Synergism

FIG. 7.-Different patterns of inhibition produced on primary plates by the diffusion at right angles of two antibiotics (diagrammatic).

shown. The only limiting factors are the original concentrations in the strips. This method has recently been used for the investigation of inhibitory substances acting on mycobacteria (King, Knox, and Woodroffe, 1953). In practice the experiment is carried out as follows:

A strip of blotting paper, measuring $5 \mathrm{~cm}$. by $0.5-1.0 \mathrm{~cm}$., is moistened evenly by placing it on blotting paper soaked in a solution of one drug. It is placed on the surface of a Petri dish containing nutrient agar previously inoculated with a young broth culture. Another strip containing the second drug is placed at right angles to it to form either a " $T$ " or a cross. The concentrations of the antibiotics used should be approximately 20 times the maximum blood concentration attained with therapeutic dosage. During subsequent incubation bacteria in the angles between the strips will be subjected to different combinations of concentrations of the two drugs. After any chosen period of incubation a note is made of the appearances of the zones of inhibition, the strips are removed and a replica plate is made.

Primary Plates (Fig. 7).--If no interaction: demonstrable by bacteriostasis has occurred, the limits of inhibition due to both drugs form a sharp₹ right angle. Antagonism is shown by the presence $\vec{\bullet}$ in the corner of a "tongue " of growth running in towards one of the strips, or, more rarely, towards the angle. This shows that a normally inhibitoryo concentration of one drug has lost its action in the presence of a sub-inhibitory concentration of the other. Experience with the replica plating method $\frac{\mathcal{Q}}{\mathbb{Q}}$ has shown that the appearance of "tongues" cor- $\cong$ relates with antagonism of lethal action duringō the first eight hours of incubation of the primary plate; this correlation may be lost during subsequent incubation. With later sampling therefore it may be found that such tongues contain no viable organisms. If there is synergism of inhibition the angle will be rounded off. This indicates. that a normally sub-inhibitory concentration of

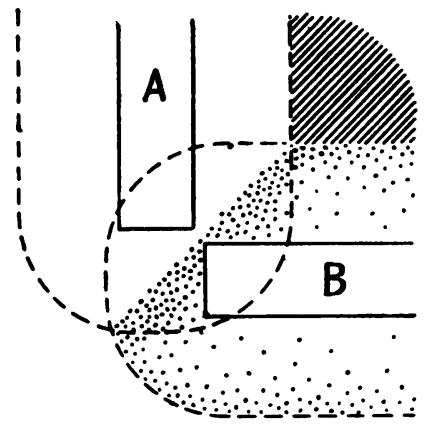

Antagouism

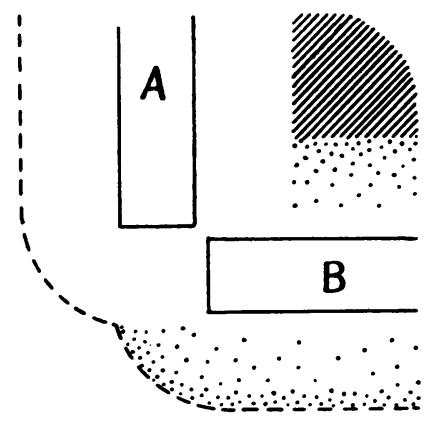

No interference

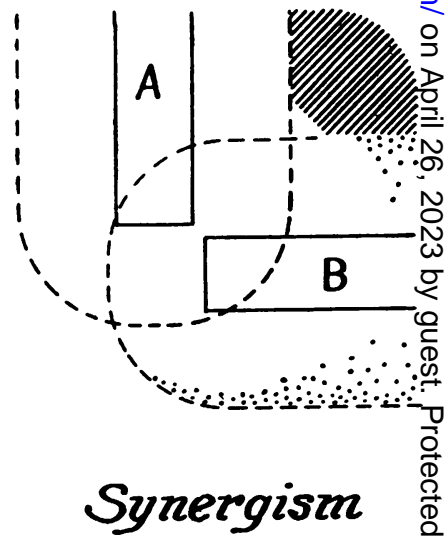

Synergism

Fio. 8.-Different patterns of lethal effect seen on replica plates (diagrammatic). 
one drug has become inhibitory in the presence of a sub-inhibitory concentration of the other.

Replica Plates.-These will reveal the bactericidal action of the combinations. The area nearest to the strip will show the effect of concentrations higher than the minimum inhibitory concentration while the outline or edge of the pattern indicates the effect of combinations near the minimum inhibitory concentration.

The possible interactions between two drugs are shown diagrammatically in Fig. 8. One drug (A) is highly lethal and the other (B) less so. Synergism is revealed by the growth of fewer survivors in the region where the organisms were subjected to the action of both drugs. The angle between the limits of lethal action will also show a rounding off, similar to that described for the primary plate. If both drugs are highly lethal this rounding off may be the only effect demonstrable. Antagonism is indicated by a greater degree of survival in the area of combined action, often accompanying a small tongue of confluent growth extending inwards from the angle between the limits of lethal effect.

All these combinations have been observed with various antibiotics, and photographs illustrating these findings are shown in Fig. 9. In the interpretation of these it must be stressed that the outline indicates the effect on the whole bacterial population, and is the result of antibiotic concentrations close to the minimum inhibitory levels. The central areas show the effects of combinations significantly higher, which are acting on a lesser population-i.e., those organisms that have survived the action just described.

Method 5: Disc Techniques.-Blotting-paper discs may be used to show interactions of antibiotics in two ways: (a) by superimposing two discs each containing a different antibiotic on the primary plate, $(b)$ by placing the discs side by
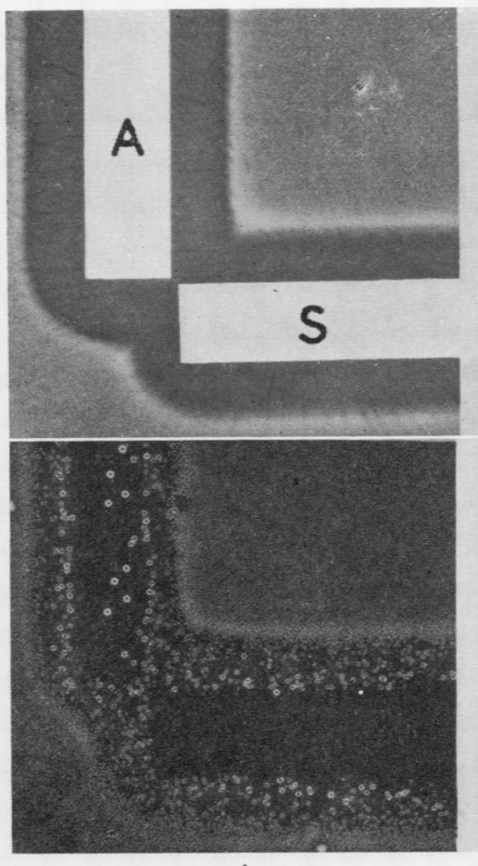

d

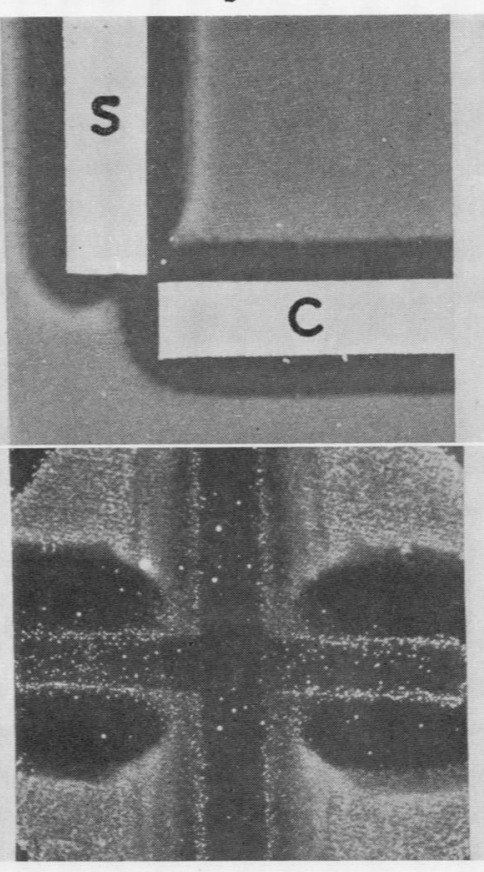

e

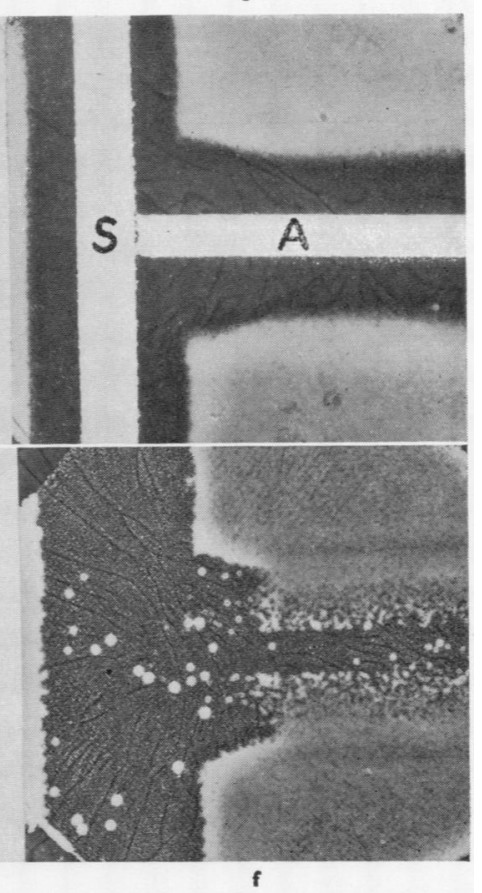

FIG. 9.-Examples of antibiotic interaction : Primary Plates (Inhibition).-(a) No interaction between aureomycin and streptomycin acting on a strain of Bact. coli. The inhibition edges form a sharp right, angle. (b) Antagonism between streptomycin and chloramphenicol acting on a coagulase-positive staphylococcus. There is a "tongue" of growth extending towards the streptomycin strip. (c) Synergism between streptomycin and aureomycin acting on a coagulase-positive staphylococcus. The zone of inhibition due to aureomycin is widened in the angle. Replica Plates (Lethal Effect).-(d) No interaction between chloramphenicol (vertical zone) and aureomycin is widened in the angle. Replica Plates (Lethal Effect).- (e) Marked antagonism between chloramphenicol (vertical) and penicillin (horizontal) acting on a coagulase-positive staphy The almost complete lethal effect of the penicillin is lost in the presence of the (horizontal) acting on a strain of Strep. faecalis. The almost complete lethal effect of the penicilin is lost in the presence of the chloramphenicol, which is itself only feebly lethal. The increased survival close to the strips is probably due to the inoculum being exposed to high concentrations while still in the lag phase, and being prevented from entering the logarithmic phase in which
there is greater susceptibility to lethal action. (f) A replica of the plate shown in (c). There is marked synergism between streptomycin and aureomycin, shown by the outward extension of the almost complete lethal action of the former where the latter is also present. The synergism is less with the higher concentrations of aureomycin close to the strip. 
side, close enough together to produce overlapping of their zones of inhibition. As a control single discs containing each antibiotic are placed on the same plates.

When the discs are superimposed the replica plates show antagonism if the survival with the combination is greater than that with the more lethal antibiotic alone. Synergism is shown by effects similar to those seen with Method 4. On the whole these disc techniques are less satisfactory than the paper-strip methods, since the range of concentrations tested is more limited.

\section{Conclusion}

The final judgment of the clinical importance of the lethal action of antibiotics must rest on comparisons between therapeutic results and laboratory findings. Experience has shown that bacterial strains, even of the same species, vary widely in their response to the inhibitory action of antibiotics; a similar variation is likely in relation to their lethal action. It is felt that the techniques outlined here offer a simple tool which may help towards a solution of these problems.

\section{Summary}

Replica plating may be used to provide a simple form of viable count, adequate for practical pure poses. Several methods are described based orep this principle and designed to reveal the bacteri $\Phi$ cidal action of drugs in vitro. The methods may find applications in antiseptic testing, and may be used to assess the lethal action of antibiotics orP individual strains isolated from patients and to $\vec{\omega}$ test for antibiotic interactions.

\section{REFERENCES}

Bliss, E. A., Warth, P. T, and Long, P. H. (1952). Bull. Johns Hopk: Hosp., 90, 149.

Burnell, J. M., and Kirby, W. M. M. (1951). Antibiot and Chemother., 1, 405.

Cates, J. E., Christie, R. V., and Garrod, L. P. (1951). Brit. med. J.? $1,653$.

Elek, S. D. (1949). Brit. J. exp. Path., 30, 484.

Fairbrother, R. W., and Martyn, G. (1951). Journal of ClinicaD Pathology, 4, 374.

Humphrey, J. H., and Lightbown, J. W. (1952). J, gen. Microbiol. $\mathbf{7}, 129$.

Hunter, T. H. (1950). J. Amer. med. Ass., 144, 524.

Jawetz, E., Gunnison, J. B., Bruff, J. B., and Coleman, V. R. (1952) J. Bact., 64, 29.

King, M. B., Knox, R., and Woodroffe, R. C. (1953). Lancet, 573.

Lederberg, J., and Lederberg, E. M. (1952). J. Bact., 63, 399.

Robbins, W. C., and Tompsett, R. (1951). Amer. J. Med., 10, 278 